\title{
Profile of Patients with Isolated Distal Deep Vein Thrombosis versus Proximal Deep Vein Thrombosis or Pulmonary Embolism: RE-COVERY DVT/PE Study
}

Sebastian Schellong, MD ${ }^{1}$ Walter Ageno, $\mathrm{MD}^{2}$ Ivan B. Casella, $\mathrm{MD}^{3}$ Kok Han Chee, $\mathrm{MD}^{4}$ Sam Schulman, MD, PhD ${ }^{5,6}$ Daniel E. Singer, MD ${ }^{7}$ Marc Desch, $\mathrm{MD}^{8}$ Wenbo Tang, $\mathrm{PhD}^{9}$ Isabelle Voccia, $\mathrm{PhD}^{10}$ Kristina Zint, $\mathrm{PhD}^{11}$ Samuel Z. Goldhaber, MD ${ }^{12}$

${ }^{1}$ Medical Department 2, Municipal Hospital Dresden, Dresden, Germany

2 Department of Medicine and Surgery, University of Insubria, Varese, Italy

${ }^{3}$ Department of Surgery, Clinics Hospital, University of São Paulo, São Paulo, Brazil

${ }^{4}$ Department of Medicine, Faculty of Medicine, University of Malaya, Kuala Lumpur, Malaysia

${ }^{5}$ Department of Medicine, Thrombosis and Atherosclerosis Research Institute and McMaster University, Hamilton, Ontario, Canada

6 Department of Obstetrics and Gynecology, I.M. Sechenov First Moscow State Medical University, Moscow, Russia

7 Division of General Internal Medicine, Massachusetts General Hospital and Harvard Medical School, Boston, Massachusetts

${ }^{8}$ CardioMetabolism Respiratory Medicine, Boehringer Ingelheim International $\mathrm{GmbH}$, Ingelheim am Rhein, Germany

${ }^{9}$ Biostatistics and Data Sciences, Boehringer Ingelheim Pharmaceuticals Inc., Ridgefield, Connecticut

${ }^{10}$ Clinical Operations, Boehringer Ingelheim Canada, Burlington, Ontario, Canada

11 Department of Epidemiology, Boehringer Ingelheim International $\mathrm{GmbH}$, Ingelheim am Rhein, Germany

${ }^{12}$ Division of Cardiovascular Medicine, Brigham and Women's Hospital and Harvard Medical School, Boston, Massachusetts
Address for correspondence Sebastian Schellong, MD, Medical Department 2, Municipal Hospital Dresden, Friedrichstraße 41, 01067 Dresden, Germany

(e-mail: sebastian.schellong@klinikum-dresden.de).

Semin Thromb Hemost 2022;48:446-458.

\author{
Abstract \\ Keywords \\ - anticoagulants \\ - venous thrombosis \\ - distal deep vein \\ thrombosis \\ - direct thrombin \\ inhibitors \\ - pulmonary embolism
}

Isolated distal deep vein thrombosis (IDDVT) is presumed to be more benign than proximal DVT (PDVT) or pulmonary embolism (PE), suggesting a need for different management approaches. This subgroup analysis of the RE-COVERY DVT/PE global, observational study investigated patient characteristics, hospitalization details, and anticoagulant therapy in patients with IDDVT in real-world settings in 34 countries enrolled from January 2016 to May 2017. Data were analyzed descriptively according to the type and location of the index venous thromboembolism (VTE): IDDVT, PDVT \pm distal DVT (DDVT), and PE \pm DVT. Of the 6,095 eligible patients, 323 with DVT located outside the lower limb and no PE were excluded. Of the remaining 5,772 patients, $17.6 \%$ had IDDVT, $39.9 \%$ had PDVT \pm DDVT, and $42.5 \%$ had PE \pm DVT. IDDVT patients were younger and had fewer risk factors for VTE than the other groups. Other comorbidities were less frequent in the IDDVT group, except for varicose veins, superficial thrombophlebitis, and venous insufficiency. IDDVT patients were less likely to be diagnosed in an emergency department (22.3 vs. $29.7 \%$ for PDVT \pm DDVT and published online May 10, 2021
Issue Theme Recent Advances in Thrombosis and Hemostasis-Part VIII; Guest Editor: Sam Schulman, MD, PhD (c) 2021. Thieme. All rights reserved. Thieme Medical Publishers, Inc., 333 Seventh Avenue, 18th Floor, New York, NY 10001, USA
DOI https://doi.org/ 10.1055/s-0041-1729169. ISSN 0094-6176. 
45.4\% for PE \pm DVT) or hospitalized for VTE (29.2 vs. 48.5\% for PDVT \pm DDVT and $75.0 \%$ for $P E \pm D V T$ ). At hospital discharge or 14 days after diagnosis (whichever was later), non-vitamin $\mathrm{K}$ antagonist oral anticoagulants were the most commonly used anticoagulants (55.6\% for IDDVT, $54.7 \%$ for PDVT \pm DDVT, and $52.8 \%$ for PE \pm DVT). Although differences in patient characteristics, risk factors, and clinical management were identified, anticoagulant treatment of IDDVT was almost equal to that of PDVT or PE. Prospective studies should investigate whether, in a global perspective, this is an appropriate use of anticoagulants.

Venous thromboembolism (VTE) may be associated with significant morbidity, risk of recurrence, and fatality from pulmonary embolism (PE). Approximately two-thirds of patients present with deep vein thrombosis (DVT) and one-third with PE. ${ }^{1}$ For those patients with proximal DVT (PDVT), anticoagulation is warranted to prevent clinical PE and postthrombotic syndrome. However, there is longstanding debate as to whether patients with isolated distal DVT (IDDVT) should be anticoagulated. ${ }^{2-6}$ The decision to anticoagulate needs to balance the potential for IDDVT to extend to PDVT and PE against the increased risk of bleeding. Prospective and retrospective management trials $\mathrm{s}^{2,7}$ and literature analyses ${ }^{4,8}$ indicate that only a minority (on average $\sim 10$ to $20 \%$ ) of patients with IDDVT are at risk of developing PDVT and/or PE if left untreated. Therefore, anticoagulation may be unnecessary. Current guidelines suggest that patients with IDDVT who do not have severe symptoms or risk factors for progression may be monitored with serial imaging rather than treated with anticoagulation. For patients with severe symptoms or with risk factors that predispose to recurrent VTE (such as active cancer, previous VTE, or inpatient status), the use of anticoagulation for at least 3 months is suggested. ${ }^{9,10}$ At present, there are no other reliable predictors that identify IDDVT patients at risk of progression to PDVT/PE.

The RE-COVERY DVT/PE study is a global prospective cohort study that aims to characterize patients who present with acute VTE in routine clinical practice. ${ }^{11}$ In the primary analysis of the overall cohort, we characterized the DVT/PE patient population and explored anticoagulant use for the treatment of acute VTE. Overall, $77 \%$ of patients received oral anticoagulants (54\% non-vitamin $\mathrm{K}$ antagonist oral anticoagulants [NOACs] and $23 \%$ vitamin $\mathrm{K}$ antagonists [VKAs]), with $20 \%$ receiving parenteral anticoagulation only; NOAC treatment was less frequent in patients with cancer, chronic renal disease, heart failure, or stroke. ${ }^{12}$

Despite current guidelines, studies have shown that anticoagulants are used in almost all patients with IDDVT. ${ }^{13-16}$ The aim of this ancillary study of RE-COVERY DVT/PE was to further investigate the characteristics, management, and anticoagulant use in patients with IDDVT in clinical practice.

\section{Methods}

\section{Study Design}

RE-COVERY DVT/PE is a multicenter, international, observational study designed with two phases. Patients with acute
DVT and/or PE were recruited from Europe, North America, Asia, the Middle East, and Latin America. The rationale and design of the study have been described elsewhere. ${ }^{11}$ To minimize the risk of potential selection bias, in the first phase of the study, investigators were encouraged to include consecutive patients with acute VTE irrespective of initial treatment. As such, each patient presenting with a VTE was approached for study enrollment, irrespective of how they were treated and managed. Assessment of a patient for study participation was within 6 months after diagnosis of the acute VTE. Patients were eligible for inclusion if they were aged $\geq 18$ years with an objective diagnosis of acute DVT and/or PE. The present subgroup analysis is based on the first phase. In the second phase, the safety and effectiveness of dabigatran and VKAs over a follow-up period of 1 year will be compared.

Study initiation required the approval of dabigatran for VTE in participating countries and a robust site feasibility process to ensure that participating sites represented the standard of care within that country. These sites included hospitals, outpatient care centers, anticoagulation clinics, and general/private practice offices. The study was performed in compliance with the protocol and the principles laid down in the Declaration of Helsinki and in accordance with the applicable sections of the guidelines for Good Clinical Practice, Good Epidemiological Practice, and Good Pharmacoepidemiology Practice, and local regulations. Written informed consent was provided by patients or their legal representatives in accordance with local regulations before entering the study.

\section{Eligibility Criteria}

Inpatients or outpatients aged $\geq 18$ years with symptomatic, objectively diagnosed, acute DVT and/or PE were eligible for inclusion. Patients were excluded only if there was a need for anticoagulation for conditions other than VTE or current participation in another clinical trial for VTE.

\section{Data Collection and Analysis}

At the baseline visit, patient characteristics, as assessed by the investigator, and anticoagulant treatment administered following objective diagnosis of VTE were recorded. Investigators also recorded information about the index VTE event, including clinical signs and symptoms of VTE; type of objective testing used for VTE (venous compression ultrasonography, venography for DVT, or other examinations deemed relevant for 
routine clinical practice for DVT diagnosis); and the resultant location, extent, and severity of venous thrombus. Baseline data were collected for the time of the index event based on assessment of the patient (e.g., patient symptom history), review of hospital/medical records, and available laboratory and diagnostic test reports. As parenteral anticoagulation with heparin or fondaparinux may have preceded treatment with oral anticoagulants, anticoagulant treatments were recorded again either at hospital discharge or 14 days after diagnosis, whichever was later. Adverse events occurring during this period were also recorded. A web-based electronic data system with secure access features captured all clinical data and site/investigator characteristics and maintained a complete electronic audit trail.

Baseline patient characteristics, details of hospitalization, and choice of anticoagulant therapy were tabulated for three groups of patients according to the type and location of their index VTE. These were distal lower extremity DVT (below the popliteal vein/trifurcation area), no iliac and/or proximal lower extremity DVT, and no PE (IDDVT only); iliac and/or proximal lower extremity DVT with or without distal lower extremity DVT, but no PE (PDVT \pm distal deep vein thrombosis [DDVT]); and any PE with or without any DVT (PE \pm DVT). Therapy at baseline and at 14 days or at discharge was recorded. The assignment of anticoagulant treatment choice for the current analysis was based on the therapy at hospital discharge or 14 days after diagnosis (whichever was later); as such, patients who received parenteral anticoagulation prior to or overlapping with oral anticoagulation were considered to be treated with the relevant oral anticoagulant. The number of patients with missing data for any parameter is included in percentage calculations and shown as a category or footnote. Due to the descriptive and exploratory nature of the study, no formal statistic testing was done. Instead, 95\% confidence intervals were calculated around means (for continuous variables) and around percentages as ClopperPearson intervals (for discrete variables). ${ }^{17}$

\section{Results}

\section{Baseline Patient Characteristics}

Patients $(N=6,194)$ were consecutively enrolled from January 2016 to May 2017, from 229 sites in 34 countries (-Table 1) across Europe (59.4\%), North America (15.9\%), the Middle East/Turkey (11.0\%), Asia (9.8\%), and Latin America (3.9\%). Of these, 6,095 patients were eligible for study entry. Ninety-nine enrolled patients were excluded owing to issues with informed consent form $(n=29)$, inclusion criteria not met $(20)$, and exclusion criteria met (4); a further 46 patients had no documented VTE treatment (24 DVT, 19 PE, and 3 DVT and PE). These 46 patients ( $0.74 \%$ of the total enrolled) could not be categorized into any of the specified treatment sets and therefore were excluded. Of the 6,095 eligible patients, 323 who had DVT located outside the lower limb and no PE, were excluded, leaving 5,772 patients in this analysis. Most of the eligible patients were enrolled at hospital study sites (either

Table 1 Countries enrolling patients in the RE-COVERY DVT/PE study (total $N=6,194$ )

\begin{tabular}{|l|l|l|l|}
\hline Region and country & Patients, $\boldsymbol{n}(\%)$ & Region and country & Patients, $\boldsymbol{n}(\%)$ \\
\hline Europe & $3,618(59.4)$ & Middle East & $668(11.0)$ \\
\hline Austria & $188(3.1)$ & Arab Emirates & $2(0.0)$ \\
\hline Belgium & $43(0.7)$ & Egypt & $13(0.2)$ \\
\hline Bulgaria & $93(1.5)$ & Lebanon & $51(0.8)$ \\
\hline Czech Republic & $255(4.2)$ & Turkey & $602(9.9)$ \\
\hline Germany & $123(2.0)$ & North America & $970(15.9)$ \\
\hline Greece & $106(1.7)$ & Canada & $319(5.2)$ \\
\hline Hungary & $264(4.3)$ & USA & $651(10.7)$ \\
\hline Italy & $297(4.9)$ & Latin America & $239(3.9)$ \\
\hline Latvia & $9(0.1)$ & Argentina & $153(2.5)$ \\
\hline The Netherlands & Brazil & $19(0.3)$ \\
\hline Poland & $54(0.9)$ & Chile & $5(0.1)$ \\
\hline Portugal & $42(0.7)$ & Colombia & $56(0.9)$ \\
\hline Romania & $57(0.9)$ & Mexico & $2(0.0)$ \\
\hline Russian Federation & $101(1.7)$ & Peru & $4(0.1)$ \\
\hline Serbia & $458(7.5)$ & Asia & $600(9.8)$ \\
\hline Slovakia & $502(8.2)$ & Malaysia & $44(0.7)$ \\
\hline Slovenia & $169(2.8)$ & Philippines & $33(0.5)$ \\
\hline United Kingdom & $23(0.4)$ & South Korea & $414(6.8)$ \\
\hline & $834(13.7)$ & Thailand & $109(1.8)$ \\
\hline
\end{tabular}


university/research hospitals [29.6\%] or other hospitals [60.6\%]) with the remainder in a clinic, practice, specialist office, or medical center (6.1\%), or in primary care (3.7\%).

Baseline demographic characteristics of all eligible patients are summarized in - Table $\mathbf{2}$. The VTE index events were IDDVT in $17.6 \%$, PDVT \pm DDVT in $39.9 \%$, and $\mathrm{PE} \pm$ DVT in $42.5 \%$. In the PDVT \pm DDVT group, all patients had "proximal" and/or iliac DVT (per study definition), 15.4\% had iliac vein DVT and $41.8 \%$ also had DDVT. Proximal DVT without iliac involvement comprised $96.6 \%$ of this group and iliac DVT without other proximal location comprised $0.4 \%$. Among patients with $\mathrm{PE} \pm \mathrm{DVT}$, the majority (64.8\%) had PE alone; the remainder had PE plus any DVT, and for these patients $(n=863)$, locations were recorded as distal in 56.7\%, proximal in $68.6 \%$, and iliac vein in $9.5 \%$ (more than one location was possible).

Mean age was shown to be youngest in the IDDVT group (-Table 2). More patients with IDDVT were employed full time, and fewer were retired, compared with the other two groups. Mean calculated creatinine clearance was highest in the IDDVT group.

Patients with Caucasian ethnicity had a higher representation in the IDDVT and PDVT \pm DDVT groups than in the $\mathrm{PE} \pm \mathrm{DVT}$ group (81.7 and $78.6 \%$ vs. $70.9 \%$, respectively). In contrast, Asian and Black or African American patients had a lower representation in the IDDVT and PDVT \pm DDVT groups than in the PE \pm DVT group (10.0 and $8.8 \%$ vs. $13.5 \%$ for Asian patients; and 1.1 and $2.3 \%$ vs. $4.2 \%$ for Black or African American patients). To explore this in more detail, 19, 16, and $7 \%$ of Caucasians, Asians, and Black or African American patients had IDDVT, compared with 41,32 , and $32 \%$ with PDVT \pm DDVT and 40,52 , and $62 \%$ with $\mathrm{PE} \pm$ DVT, respectively. The ratio of IDDVT to PDVT was, therefore, relatively similar in patients who were Caucasian (0.46) and Asian (0.50), but notably lower in Black or African American patients (0.20).

Table 2 Baseline demographic characteristics

\begin{tabular}{|c|c|c|c|c|}
\hline & $\begin{array}{l}\text { Group A } \\
\text { IDDVT }^{\mathrm{a}} \\
N=1,016\end{array}$ & 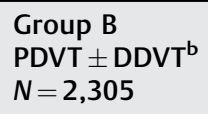 & $\begin{array}{l}\text { Group C } \\
\mathrm{PE} \pm \text { any DVT } \\
N=2,451\end{array}$ & $\begin{array}{l}\text { Total } \\
N=5,772\end{array}$ \\
\hline Age, $y ;$ mean $\pm S D$ & $\begin{array}{l}58.2 \pm 17.5 \\
{[57.1-59.3]}\end{array}$ & $\begin{array}{l}61.8 \pm 17.0 \\
{[61.1-62.5]}\end{array}$ & $\begin{array}{l}63.0 \pm 16.5 \\
{[62.4-63.7]}\end{array}$ & $\begin{array}{l}61.7 \pm 17.0 \\
Y Y\end{array}$ \\
\hline \multicolumn{5}{|l|}{ Age group, $n(\%)$} \\
\hline$<65 y$ & $\begin{array}{l}603(59.4) \\
{[56.3-62.4]}\end{array}$ & $\begin{array}{l}1182(51.3) \\
{[49.2-53.3]}\end{array}$ & $\begin{array}{l}1190(48.6) \\
{[46.6-50.6]}\end{array}$ & $\begin{array}{l}2975(51.5) \\
Y Y\end{array}$ \\
\hline 65 to $<75$ y & $\begin{array}{l}211(20.8) \\
{[18.3-23.4]}\end{array}$ & $\begin{array}{l}542(23.5) \\
{[21.8-25.3]}\end{array}$ & $\begin{array}{l}575(23.5) \\
{[21.8-25.2]}\end{array}$ & $1,328(23.0)$ \\
\hline$\geq 75 y$ & $\begin{array}{l}202(19.9) \\
{[17.5-22.5]}\end{array}$ & $\begin{array}{l}581(25.2) \\
{[23.4-27.0]}\end{array}$ & $\begin{array}{l}686(28.0) \\
{[26.2-29.8]}\end{array}$ & $\begin{array}{l}1,469(25.5) \\
Y Y\end{array}$ \\
\hline Male, ${ }^{c} n(\%)$ & $\begin{array}{l}496(48.8) \\
{[45.7-51.9]}\end{array}$ & $\begin{array}{l}1,221(53.0) \\
{[50.9-55.0]}\end{array}$ & $\begin{array}{l}1,208(49.3) \\
{[47.3-51.3]}\end{array}$ & $2,925(50.7)$ \\
\hline \multicolumn{5}{|l|}{ Ethnicity, $n(\%)$} \\
\hline White & $\begin{array}{l}830(81.7) \\
{[78.2-84.0]}\end{array}$ & $\begin{array}{l}1,812(78.6) \\
{[76.9-80.3]}\end{array}$ & $\begin{array}{l}1,737(70.9) \\
{[69.0-72.7]}\end{array}$ & $\begin{array}{l}4,379(75.9) \\
Y\end{array}$ \\
\hline Asian & $\begin{array}{l}102(10.0) \\
{[8.3-12.1]}\end{array}$ & $\begin{array}{l}202(8.8) \\
{[7.6-10.0]}\end{array}$ & $\begin{array}{l}330(13.5) \\
{[12.1-14.9]}\end{array}$ & $634(11.0)$ \\
\hline Black or African American & $\begin{array}{l}11(1.1) \\
{[0.5-1.9]}\end{array}$ & $\begin{array}{l}54(2.3) \\
{[1.8-3.0]}\end{array}$ & $\begin{array}{l}104(4.2) \\
{[3.5-5.1]}\end{array}$ & $\begin{array}{l}169(2.9) \\
Y\end{array}$ \\
\hline Unknown & $\begin{array}{l}72(7.1) \\
{[5.6-8.8]}\end{array}$ & $\begin{array}{l}228(9.9) \\
{[8.7-11.2]}\end{array}$ & $\begin{array}{l}264(10.8) \\
{[9.6-12.1]}\end{array}$ & $\begin{array}{l}564(9.9) \\
Y\end{array}$ \\
\hline Other $^{\mathrm{d}}$ & $\begin{array}{l}1(0.1) \\
{[0.00-0.05]}\end{array}$ & $\begin{array}{l}9(0.4) \\
{[0.2-0.7]}\end{array}$ & $\begin{array}{l}16(0.7) \\
{[0.4-1.1]}\end{array}$ & $26(0.5)$ \\
\hline $\mathrm{CrCl},{ }^{\mathrm{e}} \mathrm{mL} / \mathrm{min}$, mean $\pm \mathrm{SD}$ & $\begin{array}{l}102.0 \pm 43.0 \\
{[98.5-105.5]}\end{array}$ & $\begin{array}{l}92.1 \pm 42.8 \\
{[89.3-94.3]}\end{array}$ & $\begin{array}{l}94.2 \pm 47.2 \\
{[92.1-96.3]}\end{array}$ & $\begin{array}{l}94.5 \pm 45.1 \\
Y Y\end{array}$ \\
\hline $\mathrm{BMI}^{\mathrm{f}} \mathrm{kg} / \mathrm{m}^{2}$, mean $\pm \mathrm{SD}$ & $\begin{array}{l}27.9 \pm 5.3 \\
{[27.5-28.3]}\end{array}$ & $\begin{array}{l}28.0 \pm 5.7 \\
{[27.7-28.3]}\end{array}$ & $\begin{array}{l}28.4 \pm 6.7 \\
{[28.1-28.7]}\end{array}$ & $28.2 \pm 6.1$ \\
\hline Weight, ${ }^{\mathrm{g}} \mathrm{kg}$, mean $\pm \mathrm{SD}$ & $\begin{array}{l}80.6 \pm 18.3 \\
{[79.4-81.8]}\end{array}$ & $\begin{array}{l}80.6 \pm 19.3 \\
{[80.0-81.5]}\end{array}$ & $\begin{array}{l}81.8 \pm 21.9 \\
{[80.9-82.7]}\end{array}$ & $81.1 \pm 20.3$ \\
\hline Height, $^{\mathrm{h}} \mathrm{cm}$, mean $\pm \mathrm{SD}$ & $\begin{array}{l}169.7 \pm 10.0 \\
{[168.0-169.4]}\end{array}$ & $\begin{array}{l}169.3 \pm 9.9 \\
{[168.9-169.8]}\end{array}$ & $\begin{array}{l}169.3 \pm 10.5 \\
{[168.8-169.8]}\end{array}$ & $169.4 \pm 10.2$ \\
\hline Prior VTE event, $n(\%)$ & $\begin{array}{l}108(10.6) \\
{[8.8-12.7]}\end{array}$ & $\begin{array}{l}289(12.5) \\
{[11.2-14.0]}\end{array}$ & $\begin{array}{l}235(9.6) \\
{[8.5-10.8]}\end{array}$ & $632(10.9)$ \\
\hline
\end{tabular}


Table 2 (Continued)

\begin{tabular}{|c|c|c|c|c|}
\hline & $\begin{array}{l}\text { Group A } \\
\text { IDDVT }^{\mathrm{a}} \\
N=1,016\end{array}$ & $\begin{array}{l}\text { Group B } \\
\text { PDVT } \pm \text { DDVT }^{\mathrm{b}} \\
N=2,305\end{array}$ & $\begin{array}{l}\text { Group C } \\
\text { PE } \pm \text { any DVT } \\
N=2,451\end{array}$ & $\begin{array}{l}\text { Total } \\
N=5,772\end{array}$ \\
\hline \multicolumn{5}{|c|}{ Smoking history, ${ }^{i} n(\%)$} \\
\hline Non-smoker & $\begin{array}{l}501(49.3) \\
{[46.2-52.4]}\end{array}$ & $\begin{array}{l}1,081(46.9) \\
{[44.8-49.0]}\end{array}$ & $\begin{array}{l}1,193(48.7) \\
{[46.7-50.7]}\end{array}$ & $2,775(48.1)$ \\
\hline Current smoker & $\begin{array}{l}180(17.7) \\
{[15.4-20.2]}\end{array}$ & $\begin{array}{l}369(16.0) \\
{[14.5-17.6]}\end{array}$ & $\begin{array}{l}330(13.5) \\
{[12.1-14.9]}\end{array}$ & $\begin{array}{l}886(15.2) \\
Y\end{array}$ \\
\hline Ex-smoker & $\begin{array}{l}103(10.1) \\
{[8.4-12.2]}\end{array}$ & $\begin{array}{l}297(12.9) \\
{[11.4-14.3]}\end{array}$ & $\begin{array}{l}411(16.8) \\
{[15.3-18.3]}\end{array}$ & $\begin{array}{l}811(14.1) \\
Y\end{array}$ \\
\hline Unknown & $\begin{array}{l}232(22.8) \\
{[20.3-25.5]}\end{array}$ & $\begin{array}{l}558(24.2) \\
{[22.5-26.0]}\end{array}$ & $\begin{array}{l}516(21.1) \\
{[19.5-22.7]}\end{array}$ & $1,306(22.6)$ \\
\hline \multicolumn{5}{|c|}{ Insurance status for medication, ${ }^{j} n(\%)$} \\
\hline Public insurance & $\begin{array}{l}535(52.7) \\
{[49.5-55.8]}\end{array}$ & $\begin{array}{l}1,152(50.0) \\
{[47.9-52.0]}\end{array}$ & $\begin{array}{l}1,059(43.2) \\
{[41.2-45.2]}\end{array}$ & $\begin{array}{l}2,746(47.6) \\
Y\end{array}$ \\
\hline Private insurance & $\begin{array}{l}41(4.0) \\
{[2.9-5.4]}\end{array}$ & $\begin{array}{l}119(5.2) \\
{[4.3-6.1]}\end{array}$ & $\begin{array}{l}164(6.7) \\
{[5.7-7.8]}\end{array}$ & $\begin{array}{l}324(5.6) \\
Y\end{array}$ \\
\hline Out-of-pocket & $\begin{array}{l}87(8.6) \\
{[6.9-10.5]}\end{array}$ & $\begin{array}{l}206(8.9) \\
{[7.8-10.2]}\end{array}$ & $\begin{array}{l}102(4.2) \\
{[3.4-5.0]}\end{array}$ & $\begin{array}{l}395(6.8) \\
Y\end{array}$ \\
\hline Multiple types & $\begin{array}{l}35(3.4) \\
{[2.4-4.8]}\end{array}$ & $\begin{array}{l}110(4.8) \\
{[3.9-5.7]}\end{array}$ & $\begin{array}{l}131(5.3) \\
{[4.5-6.3]}\end{array}$ & $276(4.8)$ \\
\hline \multicolumn{5}{|c|}{ Employment status, ${ }^{\mathrm{k}} \mathrm{n}(\%)$} \\
\hline Retired & $\begin{array}{l}275(27.1) \\
{[24.4-29.9]}\end{array}$ & $\begin{array}{l}833(36.1) \\
{[34.2-38.1]}\end{array}$ & $\begin{array}{l}802(32.7) \\
{[30.9-34.6]}\end{array}$ & $\begin{array}{l}1,910(33.1) \\
Y Y\end{array}$ \\
\hline Works full time & $\begin{array}{l}306(30.1) \\
{[27.3-33.0]}\end{array}$ & $\begin{array}{l}534(23.2) \\
{[21.5-24.9]}\end{array}$ & $\begin{array}{l}509(20.8) \\
{[19.2-22.4]}\end{array}$ & $\begin{array}{l}1,349(23.4) \\
Y Y\end{array}$ \\
\hline Unemployed & $\begin{array}{l}87(8.6) \\
{[6.9-10.5]}\end{array}$ & $\begin{array}{l}222(9.6) \\
{[8.5-10.9]}\end{array}$ & $\begin{array}{l}298(12.2) \\
{[10.9-13.5]}\end{array}$ & $\begin{array}{l}607(10.5) \\
Y\end{array}$ \\
\hline Disabled & $\begin{array}{l}12(1.2) \\
{[0.6-2.1]}\end{array}$ & $\begin{array}{l}122(5.3) \\
{[4.4-6.3]}\end{array}$ & $\begin{array}{l}83(3.4) \\
{[2.7-4.2]}\end{array}$ & $\begin{array}{l}217(3.8) \\
Y Y\end{array}$ \\
\hline Self-employed & $\begin{array}{l}27(2.7) \\
{[1.3-3.8]}\end{array}$ & $\begin{array}{l}42(1.8) \\
{[1.3-2.5]}\end{array}$ & $\begin{array}{l}54(2.2) \\
{[1.7-2.9]}\end{array}$ & $123(2.1)$ \\
\hline Works part-time & $\begin{array}{l}24(2.4) \\
{[1.5-3.5]}\end{array}$ & $\begin{array}{l}48(2.1) \\
{[1.5-2.8]}\end{array}$ & $\begin{array}{l}38(1.6) \\
{[1.1-2.1]}\end{array}$ & $110(1.9)$ \\
\hline Other & $\begin{array}{l}19(1.9) \\
{[1.1-2.9]}\end{array}$ & $\begin{array}{l}59(2.6) \\
{[2.0-3.3]}\end{array}$ & $\begin{array}{l}57(2.3) \\
{[1.8-3.0]}\end{array}$ & $135(2.3)$ \\
\hline Unknown & $\begin{array}{l}270(26.6) \\
{[23.9-29.4]}\end{array}$ & $\begin{array}{l}488(21.2) \\
{[19.5-22.9]}\end{array}$ & $\begin{array}{l}634(25.9) \\
{[24.1-27.6]}\end{array}$ & $1,392(24.1)$ \\
\hline
\end{tabular}

Abbreviations: BMI, body mass index; $\mathrm{CrCl}$, creatinine clearance; DDVT, distal DVT; DVT, deep vein thrombosis; IDDVT, isolated DDVT; PDVT, proximal DVT; PE, pulmonary embolism; SD, standard deviation; VTE, venous thromboembolism.

Notes: Values in square brackets are $95 \%$ confidence intervals. Bold font indicates $95 \%$ confidence intervals which had no overlap between group $\mathrm{A}$ and either group B or group C or both; $\mathrm{Y}$ indicates no overlap with one other group; YY indicates no overlap with two other groups.

${ }^{a}$ No iliac vein or proximal lower limb DVT, and no PE.

${ }^{\mathrm{b}}$ lliac vein or proximal lower limb DVT (popliteal vein and above), but no PE.

${ }^{\mathrm{c}}$ Missing sex data for one patient in group $\mathrm{C}$.

${ }^{\mathrm{d}}$ Includes 18 patients with multiple answers, four who were American Indian or Alaskan Native, and four who were Native Hawaiian or other Pacific Islander.

${ }^{\mathrm{e}}$ Missing $\mathrm{CrCl}$ data for 436 patients in group A, 801 in group B, and 554 in group C.

${ }^{f}$ Missing BMI data for 213 patients in group A, 488 in group B, and 557 in group C.

${ }^{9}$ Missing weight data for 155 patients in group $A, 338$ in group $B$, and 377 in group $C$.

${ }^{h}$ Missing height data for 210 patients in group A, 482 in group B, and 546 in group C.

iMissing smoking status data for one patient in group $C$.

${ }^{\mathrm{j}}$ Missing insurance status data for 318 patients in group A, 718 in group B, and 995 in group C.

kPatients could have more than one employment status. 
Table 3 Management considerations

\begin{tabular}{|c|c|c|c|c|}
\hline & $\begin{array}{l}\text { Group A } \\
\text { IDDVT }^{\mathrm{a}} \\
N=1,016\end{array}$ & $\begin{array}{l}\text { Group B } \\
\text { PDVT } \pm \text { DDVT }^{b} \\
N=2,305\end{array}$ & $\begin{array}{l}\text { Group C } \\
\text { PE } \pm \text { any DVT } \\
N=2,451\end{array}$ & $\begin{array}{l}\text { Total } \\
N=5,772\end{array}$ \\
\hline Initial assessment of VTE in ED, ${ }^{\mathrm{c}} n(\%)$ & $\begin{array}{l}315(31.0) \\
{[28.2-33.9]}\end{array}$ & $\begin{array}{l}993(43.1) \\
{[41.0-45.1]}\end{array}$ & $\begin{array}{l}1,491(60.8) \\
{[58.9-62.8]}\end{array}$ & $\begin{array}{l}2,799(48.5) \\
\text { YY }\end{array}$ \\
\hline \multicolumn{5}{|l|}{ Diagnosis setting, ${ }^{\mathrm{d}} n(\%)$} \\
\hline Hospital & $\begin{array}{l}385(37.9) \\
{[34.9-41.0]}\end{array}$ & $\begin{array}{l}979(42.5) \\
{[40.4-44.5]}\end{array}$ & $\begin{array}{l}1,075(43.9) \\
{[41.9-45.9]}\end{array}$ & $\begin{array}{l}2,439(42.3) \\
Y\end{array}$ \\
\hline ED & $\begin{array}{l}227(22.3) \\
{[19.8-25.0]}\end{array}$ & $\begin{array}{l}685(29.7) \\
{[27.9-31.6]}\end{array}$ & $\begin{array}{l}1,112(45.4) \\
{[43.4-47.4]}\end{array}$ & $\begin{array}{l}2,024(35.1) \\
Y Y\end{array}$ \\
\hline Outpatient care center/clinic & $\begin{array}{l}238(23.4) \\
{[20.9-26.2]}\end{array}$ & $\begin{array}{l}431(18.7) \\
{[17.1-20.4]}\end{array}$ & $\begin{array}{l}208(8.5) \\
{[7.4-9.7]}\end{array}$ & $\begin{array}{l}877(15.2) \\
Y Y\end{array}$ \\
\hline Private practice office & $\begin{array}{l}165(16.2) \\
{[14.0-18.7]}\end{array}$ & $\begin{array}{l}206(8.9) \\
{[7.8-10.2]}\end{array}$ & $\begin{array}{l}56(2.3) \\
{[1.7-3.0]}\end{array}$ & $\begin{array}{l}427(7.4) \\
Y Y\end{array}$ \\
\hline Admitted to hospital, ${ }^{\mathrm{e}} \mathrm{n}(\%)$ & $\begin{array}{l}297(29.2) \\
{[26.4-32.1]}\end{array}$ & $\begin{array}{l}1,118(48.5) \\
{[46.4-50.6]}\end{array}$ & $\begin{array}{l}1,839(75.0) \\
{[73.3-76.7]}\end{array}$ & $\begin{array}{l}3,254(56.4) \\
Y Y\end{array}$ \\
\hline Treated in specialist care unit, ${ }^{f} n(\%)$ & $\begin{array}{l}19(1.9) \\
{[1.1-2.9]}\end{array}$ & $\begin{array}{l}82(3.6) \\
{[2.8-4.4]}\end{array}$ & $\begin{array}{l}461(18.8) \\
{[17.3-20.4]}\end{array}$ & $\begin{array}{l}562(9.7) \\
Y\end{array}$ \\
\hline
\end{tabular}

Abbreviations: DDVT, distal DVT; DVT, deep vein thrombosis; ED, emergency department; IDDVT, isolated DDVT; PDVT, proximal DVT; PE, pulmonary embolism; VTE, venous thromboembolism.

Notes: Values in square brackets are $95 \%$ confidence intervals. Bold font indicates $95 \%$ confidence intervals which had no overlap between group A and either group B or group C or both; $\mathrm{Y}$ indicates no overlap with one other group; $\mathrm{YY}$ indicates no overlap with two other groups.

${ }^{a}$ No iliac vein or proximal lower limb DVT, and no PE.

blliac vein or proximal lower limb DVT (popliteal vein and above), but no PE.

'Data missing for one patient in group A and three in group B.

${ }^{\mathrm{d}}$ Data missing for one patient in group $A$ and four in group $B$.

e Data missing for one patient in group $A$ and three in group $B$.

fData missing for 267 patients in group A, 524 in group B, and 479 in group C.

\section{Clinical Management Considerations}

The likelihood of having initial assessment of VTE in an emergency department increased with the seriousness of the index event (31.0\% in the IDDVT group vs. 43.1 and $60.8 \%$ in the PDVT \pm DDVT and PE \pm DVT groups, respectively), as did the likelihood of being diagnosed in an emergency department $(22.3,29.7$, and $45.4 \%$, respectively), and the likelihood of being admitted to hospital for VTE (29.2, 48.5, and $75.0 \%$, respectively; - Table 3 ).

- Table 4 summarizes the DVT symptoms and investigations. Investigators reported leg tenderness/pain in approximately $78 \%$ of patients with IDDVT or PDVT \pm DDVT, compared with $50 \%$ in the PE \pm DVT group. Other symptoms were less common in the IDDVT group than in the PDVT \pm DDVT group: leg swelling, 74.1 versus $87.5 \%$; skin warmth, 17.6 versus $23.8 \%$; and skin discoloration, 18.0 versus $23.5 \%$, respectively. Venous ultrasonography was used for DVT examination in $90.6 \%$ of patients, with $2.2 \%$ examined using conventional venography and $6.2 \%$ using other methods. For investigation of $\mathrm{PE}$, chest computed tomographic scans were performed in $69.8 \%$, pulmonary angiography in $28.9 \%$, ventilation/perfusion lung scan in $10.0 \%$, and other examination in $16.7 \%$ of patients.

\section{VTE Risk Factors and Comorbidities}

Selected clinical features (comorbidity and/or medical history) that might be considered as risk factors for VTE are shown in - Fig. 1. Patients with IDDVT had a history of VTE in $10.6 \%$ of cases, compared with $12.5 \%$ in the PDVT \pm DDVT group and $9.6 \%$ in the PE \pm DVT group. The prevalence of several of the other risk factors was lower in the IDDVT group than in the PDVT \pm DDVT and PE \pm DVT groups. For example, cancer was reported in $7.5,10.2$, and $12.1 \%$ of patients with IDDVT, PDVT \pm DDVT, and PE \pm DVT, respectively. Approximately $6 \%$ of the IDDVT and PDVT \pm DDVT groups had trauma or surgery compared with $8 \%$ of the PE \pm DVT group. An exception to this trend was immobilization, which was reported for $4.0 \%$ of the IDDVT group, but 2.8 and $1.9 \%$ of patients with PDVT \pm DDVT and $\mathrm{PE} \pm \mathrm{DVT}$, respectively.

-Figure 2 shows the most frequently reported other comorbidities at the baseline visit. Typically, these were also more prevalent in the patients with PDVT \pm DDVT or with $\mathrm{PE} \pm$ DVT compared with the IDDVT group (e.g., hypertension, diabetes mellitus, coronary artery disease, heart failure, and myocardial infarction). Varicose veins were more common in the IDDVT group (5.1\%) than in the PDVT \pm DDVT $(3.2 \%)$ and PE \pm DVT (2.1\%) groups. Superficial thrombophlebitis and venous insufficiency were also found more frequently in patients with DVT than in the group with $\mathrm{PE} \pm \mathrm{DVT}$.

\section{Anticoagulant Treatments}

More than half of the patients with IDDVT (55.6\%), PDVT \pm DDVT (54.7\%), and PE \pm DVT (52.8\%) were treated with NOACs (-Fig. 3). VKAs were the next most frequently prescribed anticoagulant option. 
Table 4 DVT symptoms and investigations

\begin{tabular}{|c|c|c|c|c|}
\hline & $\begin{array}{l}\text { Group A } \\
\text { IDDVT }^{\mathrm{a}}\end{array}$ & 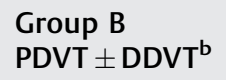 & $\begin{array}{l}\text { Group C } \\
\mathrm{PE} \pm \text { any DVT }\end{array}$ & Total \\
\hline Patients with DVT, $n$ (\%) & $1,016(100)$ & $2,305(100)$ & $863(100)$ & $4,184(100)$ \\
\hline Leg tenderness/pain, ${ }^{c} n(\%)$ & $\begin{array}{l}795(78.2) \\
{[75.6-80.7]}\end{array}$ & $\begin{array}{l}1,796(77.9) \\
{[76.2-79.6]}\end{array}$ & $\begin{array}{l}428(49.6) \\
{[46.2-53.0]}\end{array}$ & $\begin{array}{l}3,019(72.2) \\
Y\end{array}$ \\
\hline Leg swelling, ${ }^{\mathrm{d}} n(\%)$ & $\begin{array}{l}753(74.1) \\
{[71.3-76.8]}\end{array}$ & $\begin{array}{l}2,016(87.5) \\
{[86.0-88.8]}\end{array}$ & $\begin{array}{l}502(58.2) \\
{[54.8-61.5]}\end{array}$ & $\begin{array}{l}3,271(78.2) \\
Y Y\end{array}$ \\
\hline Skin warmth, ${ }^{e} n(\%)$ & $\begin{array}{l}179(17.6) \\
{[15.3-20.1]}\end{array}$ & $\begin{array}{l}549(23.8) \\
{[22.1-25.6]}\end{array}$ & $\begin{array}{l}115(13.3) \\
{[11.1-15.8]}\end{array}$ & $\begin{array}{l}843(20.1) \\
Y Y\end{array}$ \\
\hline Skin discoloration, ${ }^{f} n(\%)$ & $\begin{array}{l}183(18.0) \\
{[15.7-20.5]}\end{array}$ & $\begin{array}{l}542(23.5) \\
{[21.8-25.3]}\end{array}$ & $\begin{array}{l}104(12.1) \\
{[11.1-15.8]}\end{array}$ & $\begin{array}{l}829(19.8) \\
Y\end{array}$ \\
\hline \multicolumn{5}{|l|}{ Type of investigation, ${ }^{9} n(\%)$} \\
\hline Venous ultrasonography & $\begin{array}{l}921(90.6) \\
{[88.7-92.4]}\end{array}$ & $\begin{array}{l}2,102(91.2) \\
{[90.0-92.3]}\end{array}$ & $\begin{array}{l}766(88.8) \\
{[86.5-90.8]}\end{array}$ & $3,789(90.6)$ \\
\hline Conventional venography & $\begin{array}{l}22(2.2) \\
{[1.4-3.3]}\end{array}$ & $\begin{array}{l}78(3.4) \\
{[2.7-4.2]}\end{array}$ & $\begin{array}{l}39(4.5) \\
{[3.2-6.1]}\end{array}$ & $139(3.3)$ \\
\hline Other & $\begin{array}{l}63(6.2) \\
{[4.8-7.9]}\end{array}$ & $\begin{array}{l}151(6.6) \\
{[5.6-7.6]}\end{array}$ & $\begin{array}{l}45(5.2) \\
{[3.8-6.9]}\end{array}$ & $259(6.2)$ \\
\hline
\end{tabular}

Abbreviations: DDVT, distal DVT; DVT, deep vein thrombosis; IDDVT, isolated DDVT; PDVT, proximal DVT; PE, pulmonary embolism.

Notes: Values in square brackets are $95 \%$ confidence intervals. Bold font indicates $95 \%$ confidence intervals which had no overlap between group $A$ and either group B or group C or both; $\mathrm{Y}$ indicates no overlap with one other group; YY indicates no overlap with two other groups.

${ }^{\mathrm{a}}$ No iliac vein or proximal lower limb DVT, and no PE.

blliac vein or proximal lower limb DVT (popliteal vein and above), but no PE.

'Data unknown for 28 patients in group A, 81 in group B, and 42 in group C.

${ }^{\mathrm{d} D a t a}$ unknown for 28 patients in group A, 58 in group B, and 29 in group C.

${ }^{e}$ Data unknown for 66 patients in group $A, 173$ in group $B$, and 60 in group $C$.

fData unknown for 71 patients in group A, 160 in group B, and 60 in group C.

${ }^{9}$ Data unknown for 19 patients in group $A, 38$ in group B, and 51 in group C.

As shown in -Table $\mathbf{5}, 19.3 \%$ of all patients received parenteral anticoagulation only during the initial treatment period (up to 14 days or hospital discharge, whichever was later). Unfractionated heparin (UFH) was administered slightly more frequently in patients with PE than in DVT alone: IDDVT (0.7\%), PDVT \pm DDVT (1.2\%), and PE \pm DVT (2.9\%). There was a similar pattern for low-molecular-weight heparin (LMWH) alone. The once-daily regimen was favored over twice daily in the IDDVT group. The percentages of patients who received parenteral therapy prior to oral anticoagulation during the initial treatment period were $48.4 \%$ for IDDVT, $53.4 \%$ for PDVT \pm DDVT, and $59.4 \%$ for PE \pm DVT. The same distribution between UFH and LMWH, and once and twice daily, respectively, was observed, as for parenteral therapy only.

\section{Discussion}

Our findings show that nearly one-fifth of patients with acute VTE presented with IDDVT alone. These patients were younger and had fewer VTE risk factors and other comorbidities than the patients with $\mathrm{PDVT} \pm \mathrm{DDVT}$ or $\mathrm{PE} \pm \mathrm{DVT}$. There were differences in clinical presentation and management settings among the groups, but only small differences in the choice of anticoagulant therapy.

Several noninterventional studies have provided data on the real-world use of anticoagulants in patients with IDDVT and PDVT. These included a single-center cohort in Italy, and respective cohorts in the international RIETE registry, the French OPTIMEV study, and the global GARFIELD-VTE registry (- Table 6). ${ }^{13-16,18}$ GARFIELD-VTE also included data on patients with $\mathrm{PE} \pm \mathrm{DVTT}^{16}$ We note that the analysis of the single-center cohort excluded patients with prior VTE, ${ }^{13}$ whereas the other studies, and RE-COVERY DVT/PE, did not apply this exclusion. For the OPTIMEV study, a separate analysis of the subgroup with a first event has been presented. ${ }^{19}$ In our study, the proportion of patients with prior VTE was 10 to $12 \%$. Despite the possibility that prior episodes of VTE may influence the composition of risk factors, comorbidities, and choices of anticoagulants, these patients were not analyzed separately due to their small number.

In all studies, patients with IDDVT were younger than those with PDVT or PE \pm DVT. The most similar study in terms of scope and observation dates was GARFIELD-VTE, involving 10,088 patients with VTE from 28 countries worldwide between 2014 and 2017. The GARFIELD-VTE study was consistent with RE-COVERY DVT/PE in finding that approximately one-fifth of patients had IDDVT, $40 \%$ had PDVT, and $40 \%$ had $\mathrm{PE} \pm \mathrm{DVT}$. In both studies, IDDVT accounted for approximately 31 to $36 \%$ of the patients with DVT (without PE). ${ }^{16}$ The proportion with IDDVT was similar in RIETE (17.3\%) and the Italian cohort study (24.3\%), but was much higher in OPTIMEV (56.8\%). Differences may be explained by differences in study settings and, mainly, by differences in ultrasound protocols. ${ }^{14,15,20}$ In France, Germany, Austria, and Switzerland, 

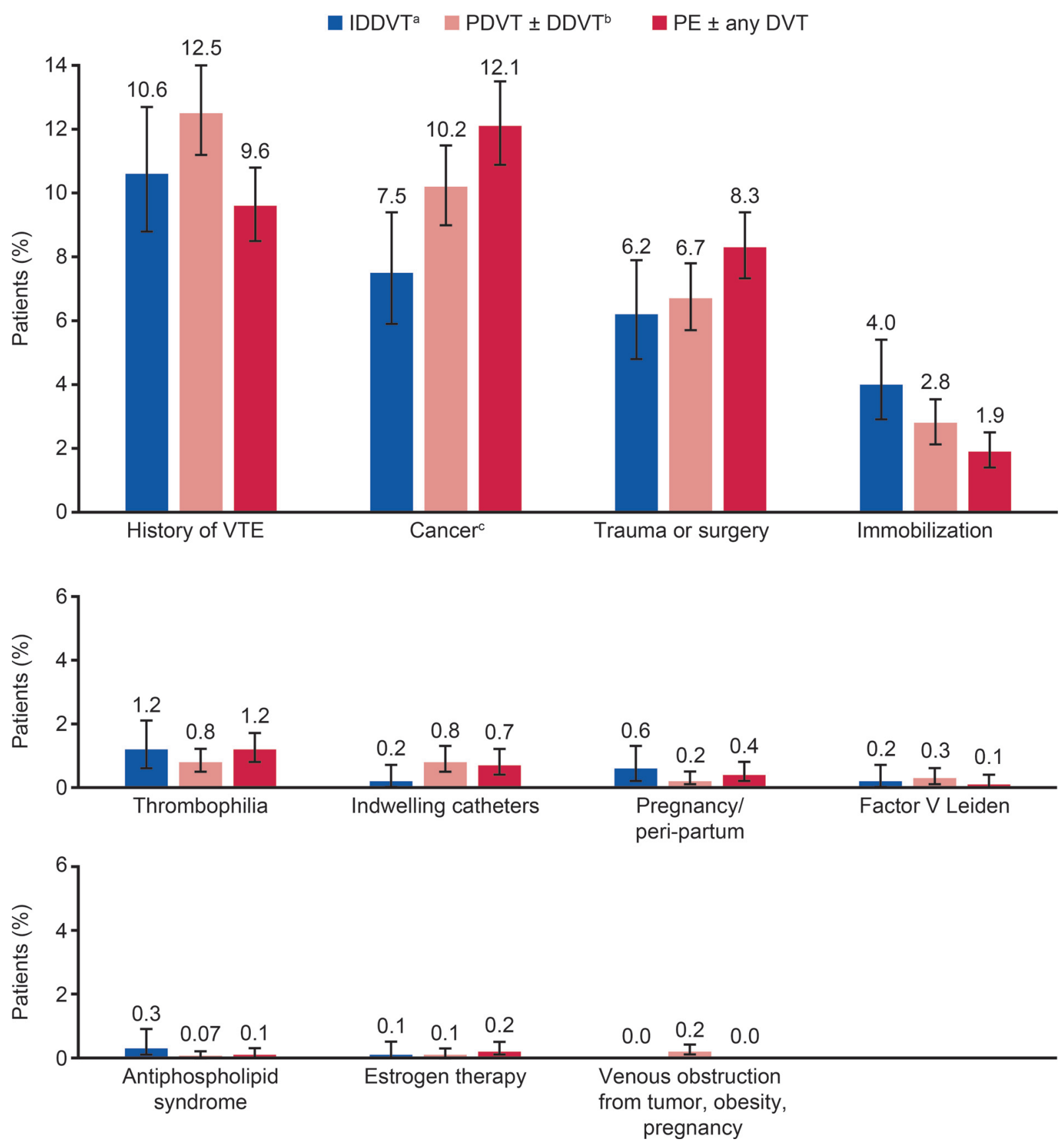

Fig. 1 Risk factors for VTE according to type and location of index VTE. Note: Based on discussion of the literature, the authors selected 11 clinical features as potential risk factors for VTE. The total numbers (\%) of patients with any of the selected risk factors were as follows: IDDVT, 268 (28.4); PDVT \pm DDVT, 688 (29.8); and PE \pm DVT, 729 (29.7). DDVT, distal DVT; DVT, deep vein thrombosis; IDDVT, isolated DDVT; PDVT, proximal DVT; PE, pulmonary embolism; VTE, venous thromboembolism. Error bars show the $95 \%$ confidence intervals. ${ }^{a}$ No iliac vein or proximal lower limb DVT, and no PE. ' Illiac vein or proximal lower limb DVT (popliteal vein and above), but no PE. 'Cancer excluding nonmelanoma skin cancer.

and to a lesser extent in Italy, almost all sonographers examine the calf veins in all patients with suspected DVT. Ultrasound protocols differ substantially across countries and even between sites within the same country. This directly influences the recorded proportion with IDDVT.

While IDDVT was detected in similar proportions of Caucasians and Asians (19 and 16\% in RE-COVERY; 21 and $22 \%$ in GARFIELD-VTE), fewer Black or African American patients had IDDVT detected (7\% in RE-COVERY DVT/PE and $11 \%$ in GARFIELD-VTE). ${ }^{16}$ This equates to a ratio of
IDDVT:PDVT of 0.46 in Caucasians and 0.50 in Asians, but 0.20 in Black or African American patients. GARFIELD-VTE showed similar results: a ratio of 0.59 in Caucasians and 0.55 in Asians, but 0.20 in Black or African American patients. These observations raise the question of detection bias due to underlying health care disparities. There might be a higher threshold to seek medical care among Black or African American patients. Variations in diagnostic testing might be another reason for the country differences observed in GARFIELD-VTE, where the ratio of IDDVT:PDVT ranged from 

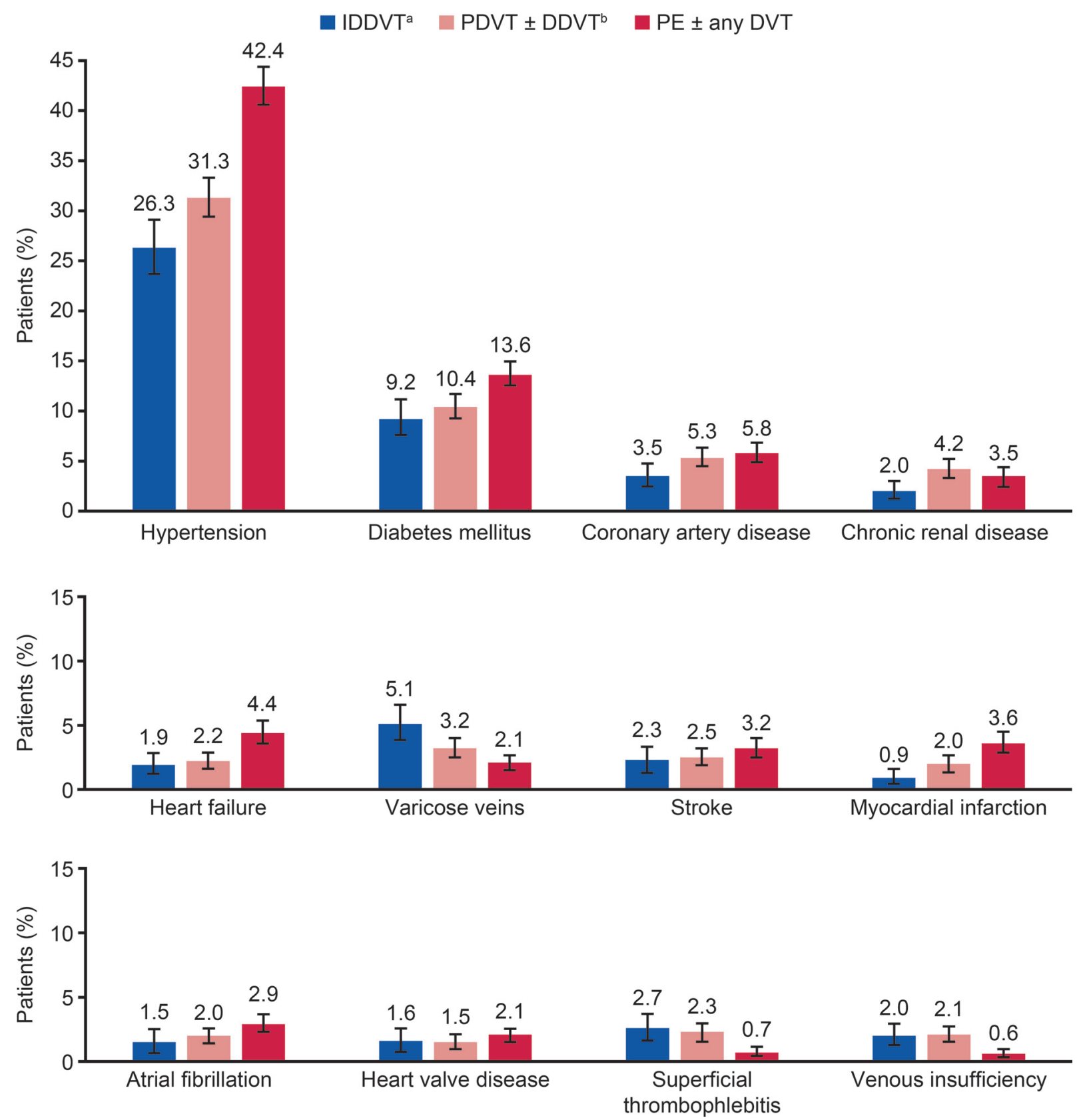

Fig. 2 Other comorbidities and/or medical history according to type and location of index VTE. Note: Other comorbidities and/or medical history include clinical features other than those selected by the authors as potential risk factors for VTE (see - Fig. 1). Comorbidities and/or medical history present in $\geq 2 \%$ of patients in any subgroup. Patients could have more than one comorbidity and/or medical history. The following were considered: atrial fibrillation, Behcet's disease, chemical phlebitis, chronic hepatic disease, chronic renal disease, coronary artery disease, diabetes mellitus, disseminated intravascular coagulation, hemolytic anemias, heart failure, heart valve disease, hypertension, inflammatory bowel disease, myocardial infarction, nephrotic syndrome, peripheral vascular disease, rheumatoid arthritis, sepsis, stroke, superficial vein thrombosis, systemic lupus erythematosus, varicose veins, venous insufficiency, and venous insufficiency or varicose veins. The total numbers (\%) of patients with any of the other comorbidities and/or medical history were as follows: IDDVT, 376 (37.0); PDVT \pm DDVT, 1,038 (45.0); and PE \pm DVT, 1,333 (54.4). DDVT, distal DVT; DVT, deep vein thrombosis; IDDVT, isolated DDVT; PDVT, proximal DVT; PE, pulmonary embolism; VTE, venous thromboembolism. Error bars show the $95 \%$ confidence intervals. ${ }^{a}$ No iliac vein or proximal lower limb DVT, and no PE. blliac vein or proximal lower limb DVT (popliteal vein and above), but no PE.

0.15 (95\% CI, 0.11-0.18) in Canada to 1.96 (95\% CI, 1.51-2.41) in Australia. ${ }^{16}$ With such wide disparities in the diagnosis of IDDVT, it is possible that certain centers/countries under- or overdiagnose IDDVT.

In our study, $60 \%$ of IDDVT patients were diagnosed in a hospital or hospital-affiliated setting (22\% in an emergency department and $38 \%$ in another hospital-associated outpa- tient facility). A smaller proportion ( $29 \%)$ of our study patients was hospitalized compared with GARFIELD-VTE, in which $61 \%$ were treated as inpatients. ${ }^{16}$ These findings highlight the heterogeneity of real-world studies. However, in both studies, IDDVT was less often treated in hospital than $\mathrm{PDVT} \pm \mathrm{DDVT}$ or $\mathrm{PE} \pm \mathrm{DVT}$, reflecting the anticipated lower severity. 


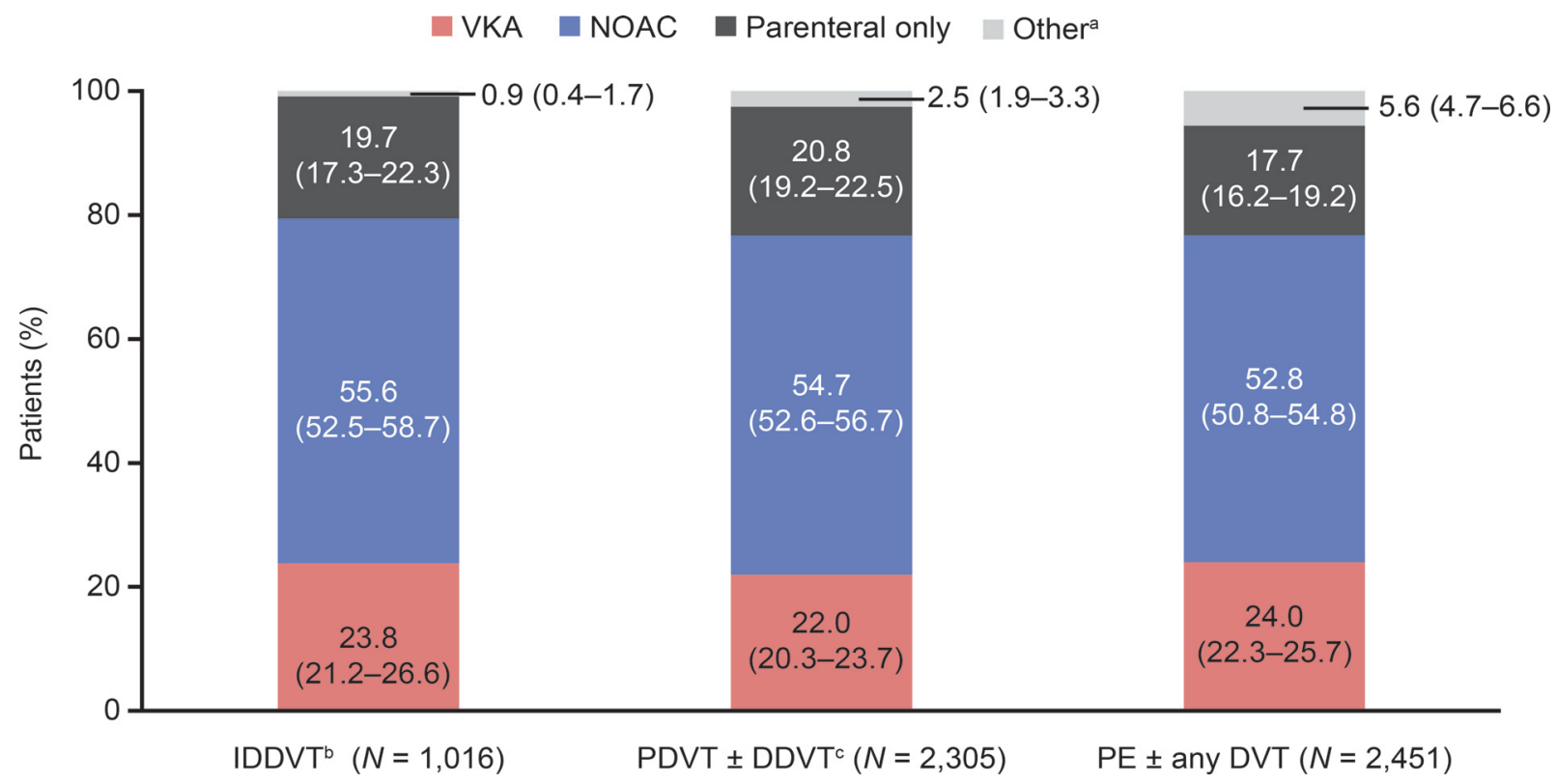

Fig. 3 Main anticoagulation therapy according to type and location of index VTE. DDVT, distal DVT; DVT, deep vein thrombosis; IDDVT, isolated DDVT; PDVT, proximal DVT; NOAC, non-VKA oral anticoagulant; PE, pulmonary embolism; VKA, vitamin K antagonist; VTE, venous thromboembolism. Values in parentheses are $95 \%$ confidence intervals. ${ }^{a}$ Other includes catheter-directed thrombolysis, systemic thrombolysis, and other treatments, respectively, as follows: 0,0 , and 9 patients in the IDDVT group; 4, 1, and 53 in the PDVT \pm DDVT group; and 4, 8 , and 125 in the PDVT \pm any DVT group. ${ }^{b}$ No iliac vein or proximal lower limb DVT, and no PE. Clliac vein or proximal lower limb DVT (popliteal vein and above), but no PE.

Table 5 Details of parenteral anticoagulation therapy given alone or prior to oral anticoagulation in the period from baseline up to hospital discharge or 14 days after diagnosis (whichever was later)

\begin{tabular}{|c|c|c|c|c|}
\hline & $\begin{array}{l}\text { Group A } \\
\text { IDDVT }^{\mathrm{a}} \\
N=1,016\end{array}$ & $\begin{array}{l}\text { Group B } \\
\text { PDVT } \pm \text { DDVT }^{b} \\
N=2,305\end{array}$ & $\begin{array}{l}\text { Group C } \\
\text { PE } \pm \text { any DVT } \\
N=2,451\end{array}$ & $\begin{array}{l}\text { Total } \\
N=5,772\end{array}$ \\
\hline \multicolumn{5}{|c|}{ Details of parenteral anticoagulation therapy alone } \\
\hline Parenteral therapy, $n(\%)$ & $\begin{array}{l}200(19.7) \\
{[17.3-22.3]}\end{array}$ & $\begin{array}{l}480(20.8) \\
{[19.2-22.5]}\end{array}$ & $\begin{array}{l}433(17.7) \\
{[16.2-19.2]}\end{array}$ & $1,113(19.3)$ \\
\hline UFH, $n(\%)$ & $\begin{array}{l}7(0.7) \\
{[0.3-1.4]}\end{array}$ & $\begin{array}{l}27(1.2) \\
{[0.8-1.7]}\end{array}$ & $\begin{array}{l}71(2.9) \\
{[2.3-3.6]}\end{array}$ & $\begin{array}{l}105(1.8) \\
Y\end{array}$ \\
\hline LMWH, $n(\%)$ & $\begin{array}{l}195(19.2) \\
{[16.8-21.8]}\end{array}$ & $\begin{array}{l}472(20.5) \\
{[18.8-22.2]}\end{array}$ & $\begin{array}{l}416(17.0) \\
{[15.5-18.5]}\end{array}$ & $1,083(18.8)$ \\
\hline \multicolumn{5}{|l|}{ Frequency $^{\mathrm{c}}$} \\
\hline QD & $\begin{array}{l}131(67.2) \\
{[60.1-73.7]}\end{array}$ & $\begin{array}{l}262(55.5) \\
{[50.9-60.1]}\end{array}$ & $\begin{array}{l}184(44.2) \\
{[39.4-49.2]}\end{array}$ & $\begin{array}{l}577(53.3) \\
Y\end{array}$ \\
\hline BID & $\begin{array}{l}64(32.8) \\
{[26.3-39.9]}\end{array}$ & $\begin{array}{l}206(43.6) \\
{[39.1-48.3]}\end{array}$ & $\begin{array}{l}224(53.8) \\
{[48.9-58.7]}\end{array}$ & $\begin{array}{l}494(45.6) \\
Y\end{array}$ \\
\hline Other or missing & 0 & $\begin{array}{l}4(0.8) \\
{[0.2-2.2]}\end{array}$ & $\begin{array}{l}8(1.9) \\
{[0.8-3.8]}\end{array}$ & $12(1.1)$ \\
\hline Fondaparinux, $n(\%)$ & $\begin{array}{l}4(0.4) \\
{[0.1-1.0]}\end{array}$ & $\begin{array}{l}9(0.4) \\
{[0.2-0.7]}\end{array}$ & $\begin{array}{l}9(0.4) \\
{[0.2-0.7]}\end{array}$ & $22(0.4)$ \\
\hline \multicolumn{5}{|c|}{ Details of parenteral anticoagulation therapy prior to oral anticoagulant therapy } \\
\hline Parenteral therapy, $n(\%)$ & $\begin{array}{l}492(48.4) \\
{[45.3-51.5]}\end{array}$ & $\begin{array}{l}1,230(53.4) \\
{[51.3-55.4]}\end{array}$ & $\begin{array}{l}1,455(59.4) \\
{[57.4-61.3]}\end{array}$ & $3,177(55.0)$ \\
\hline $\mathrm{UFH}, n(\%)$ & $\begin{array}{l}58(5.7) \\
{[4.4-7.3]}\end{array}$ & $\begin{array}{l}322(14.0) \\
{[12.6-15.5]}\end{array}$ & $\begin{array}{l}380(15.5) \\
{[14.1-17.0]}\end{array}$ & $760(13.2)$ \\
\hline LMWH, $n(\%)$ & $\begin{array}{l}435(42.8) \\
{[39.7-45.9]}\end{array}$ & $\begin{array}{l}925(40.1) \\
{[38.1-42.2]}\end{array}$ & $\begin{array}{l}1,203(49.1) \\
{[47.1-51.1]}\end{array}$ & $2,563(44.4)$ \\
\hline
\end{tabular}

(Continued) 
Table 5 (Continued)

\begin{tabular}{|c|c|c|c|c|}
\hline & $\begin{array}{l}\text { Group A } \\
\text { IDDVT }^{\mathrm{a}} \\
N=1,016\end{array}$ & 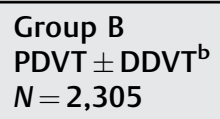 & $\begin{array}{l}\text { Group C } \\
\text { PE } \pm \text { any DVT } \\
N=2,451\end{array}$ & $\begin{array}{l}\text { Total } \\
N=5,772\end{array}$ \\
\hline \multicolumn{5}{|l|}{ Frequency $^{c}$} \\
\hline QD & $\begin{array}{l}215(49.4) \\
{[44.6-54.2]}\end{array}$ & $\begin{array}{l}366(39.6) \\
{[36.4-42.8]}\end{array}$ & $\begin{array}{l}361(30.0) \\
{[27.4-32.7]}\end{array}$ & $\begin{array}{l}942(36.8) \\
Y Y\end{array}$ \\
\hline BID & $\begin{array}{l}211(48.5) \\
{[43.7-53.3]}\end{array}$ & $\begin{array}{l}540(58.4) \\
{[55.1-61.6]}\end{array}$ & $\begin{array}{l}809(67.2) \\
{[64.5-69.9]}\end{array}$ & $\begin{array}{l}1,560(60.9) \\
Y Y\end{array}$ \\
\hline Other or missing & $\begin{array}{l}4(0.9) \\
{[0.3-2.3]}\end{array}$ & $\begin{array}{l}9(1.0) \\
{[0.4-1.8]}\end{array}$ & $\begin{array}{l}9(0.7) \\
{[0.3-1.4]}\end{array}$ & $22(0.4)$ \\
\hline Fondaparinux, $n(\%)$ & $\begin{array}{l}8(0.8) \\
{[0.3-1.5]}\end{array}$ & $\begin{array}{l}25(1.1) \\
{[0.7-1.6]}\end{array}$ & $\begin{array}{l}39(1.6) \\
{[1.2-2.3]}\end{array}$ & $72(1.2)$ \\
\hline
\end{tabular}

Abbreviations: BID, twice daily; DDVT, distal DVT; DVT, deep vein thrombosis; IDDVT, isolated DDVT; LMWH, low-molecular-weight heparin; PDVT, proximal DVT; PE, pulmonary embolism; QD, once daily; UFH, unfractionated heparin.

Notes: Values in square brackets are $95 \%$ confidence intervals. Bold font indicates $95 \%$ confidence intervals which had no overlap between group $\mathrm{A}$ and either group B or group C or both; $\mathrm{Y}$ indicates no overlap with one other group; YY indicates no overlap with two other groups.

${ }^{a}$ No iliac vein or proximal lower limb DVT, and no PE.

blliac vein or proximal lower limb DVT (popliteal vein and above), but no PE.

${ }^{c}$ Percentages were calculated based on the number of patients who received the respective therapy.

Table 6 Demographics, risk factors, and anticoagulation for IDDVT, PDVT \pm DDVT, and PE \pm any DVT, reported in observational VTE studies

\begin{tabular}{|c|c|c|c|c|c|c|c|c|c|c|c|c|}
\hline & \multicolumn{2}{|c|}{$\begin{array}{l}\text { Barco et al }{ }^{13} \\
\text { Single-center, } \\
\text { retrospective } \\
N=831 \\
\text { Italy, } \\
2000-2012\end{array}$} & \multicolumn{2}{|c|}{$\begin{array}{l}\text { RIETE, }{ }^{14} \\
N=11,086 \\
24 \text { countries } \\
\text { worldwide } \\
2001-2008\end{array}$} & \multicolumn{2}{|c|}{$\begin{array}{l}\text { OPTIMEV, }{ }^{15} \\
N=1,643 \\
\text { France, } \\
2004-2006\end{array}$} & \multicolumn{3}{|c|}{$\begin{array}{l}\text { GARFIELD-VTE, }{ }^{16} \\
N=10,088 \\
28 \text { countries } \\
\text { worldwide } \\
2014-2017\end{array}$} & \multicolumn{3}{|c|}{$\begin{array}{l}\text { RE-COVERY DVT/PE, } \\
N=5,722 \\
34 \text { countries } \\
\text { worldwide } \\
2016-2017\end{array}$} \\
\hline & IDDVT & PDVT & IDDVT & PDVT & IDDVT & PDVT & IDDVT & PDVT & PE & IDDVT & PDVT & $\mathrm{PE}$ \\
\hline Patients, $n(\%)$ & $\begin{array}{l}202 \\
(24.3)\end{array}$ & $\begin{array}{l}629 \\
(75.7)\end{array}$ & $\begin{array}{l}1,921 \\
(17.3)\end{array}$ & $\begin{array}{l}9,165 \\
(82.7)\end{array}$ & $\begin{array}{l}933 \\
(56.8)\end{array}$ & $\begin{array}{l}710 \\
(43.2)\end{array}$ & $\begin{array}{l}2,145 \\
(21.3)\end{array}$ & $\begin{array}{l}3,846 \\
(38.1)\end{array}$ & $\begin{array}{l}4,097 \\
(40.6) \\
\end{array}$ & $\begin{array}{l}1,016 \\
(17.6) \\
\end{array}$ & $\begin{array}{l}2,305 \\
(39.9) \\
\end{array}$ & $\begin{array}{l}2,415 \\
(42.5) \\
\end{array}$ \\
\hline Female, \% & 56 & 49 & 49 & 48 & 58 & 52 & 52 & 49 & 49 & 51 & 47 & 51 \\
\hline $\begin{array}{l}\text { Age, mean or } \\
\text { median, years }\end{array}$ & 66 & 67 & 65 & 70 & 62 & 69 & 56 & 58 & 60 & 58 & 62 & 63 \\
\hline Caucasian ethnicity, \% & - & - & - & - & - & - & 69.1 & 64.1 & 73.6 & 81.7 & 78.6 & 70.9 \\
\hline \multicolumn{13}{|l|}{ Risk factors, \% } \\
\hline History of VTE & $3^{a}$ & $5^{a}$ & 15 & 17 & 29 & 34 & 14 & 17 & 15 & 11 & 13 & 10 \\
\hline Active cancer & 24 & 23 & 14 & 22 & 11 & 20 & 7 & 10 & 10 & 8 & 10 & 12 \\
\hline Trauma/surgery & $14 / 21$ & $9 / 17$ & $-/ 15$ & $-/ 11$ & -122 & $-/ 12$ & $13 / 15$ & $9 / 11$ & $5 / 13$ & $\begin{array}{l}6 \\
\text { (either) }\end{array}$ & $\begin{array}{l}7 \\
\text { (either) }\end{array}$ & $\begin{array}{l}8 \\
\text { (either) }\end{array}$ \\
\hline Immobilization & 23 & 17 & 24 & 28 & 18 & 20 & - & - & - & 4 & 3 & 2 \\
\hline \multicolumn{13}{|c|}{ Anticoagulation therapy, \% } \\
\hline Any anticoagulant & 97 & 88 & $97^{\mathrm{b}}$ & $97^{b}$ & $81^{c}$ & $92^{c}$ & $97^{d}$ & $98^{d}$ & $98^{d}$ & - & - & - \\
\hline OAC & 32 & 73 & - & - & - & - & - & - & - & - & - & - \\
\hline VKA & - & - & - & - & $70^{c}$ & $70^{c}$ & $24^{\mathrm{d}}$ & $31^{d}$ & $30^{\mathrm{d}}$ & $24^{\mathrm{e}}$ & $22^{\mathrm{e}}$ & $24^{\mathrm{e}}$ \\
\hline NOAC & - & - & - & - & - & - & $50^{d}$ & $46^{d}$ & $47^{d}$ & $56^{e}$ & $55^{\mathrm{e}}$ & $53^{e}$ \\
\hline Parenteral only & - & - & - & - & - & - & $17^{d}$ & $16^{\mathrm{d}}$ & $16^{\mathrm{d}}$ & $20^{\mathrm{e}}$ & $21^{\mathrm{e}}$ & $18^{\mathrm{e}}$ \\
\hline
\end{tabular}

Abbreviations: DDVT, distal DVT; DVT, deep vein thrombosis; GARFIELD-VTE: The Global Anticoagulant Registry in the FIELD-Venous Thromboembolic Events; IDDVT, isolated DDVT; NOAC, non-VKA oral anticoagulant; OAC, oral anticoagulant; OPTIMEV: OPTimisation de I'Interrogatoire dans l'évaluation du risque throMbo-Embolique Veineux; PDVT, proximal DVT; PE, pulmonary embolism; RIETE: Registro Informatizado de pacientes con Enfermedad TromboEmbólica; VKA, vitamin K antagonist; VTE, venous thromboembolism.

${ }^{\mathrm{a}}$ Family history of VTE.

${ }^{\mathrm{b}}$ For $\geq 10$ days.

${ }^{\mathrm{c}}$ For 3-month follow-up period.

${ }^{\mathrm{d}}$ For $\leq 30$ days.

eUp to hospital discharge or 14 days after diagnosis (whichever was later). 
IDDVT is associated with transient risk factors such as hospitalization and pregnancy/postpartum, while PDVT is associated more with chronic risk factors and systemic disease such as history of VTE and active cancer. ${ }^{21}$ Differing risk factor profiles for IDDVT and PDVT are presented in -Table 6. Similar to our data, RIETE, OPTIMEV, and GARFIELD-VTE all showed that lower proportions of patients with IDDVT had active cancer or a history of VTE compared with the PDVT group. ${ }^{14-16}$

Similar to RE-COVERY DVT/PE, there were relatively small differences in the choice of anticoagulant for IDDVT versus PDVT in GARFIELD-VTE. ${ }^{16,22}$ However, Barco et al reported that 32 and $65 \%$ of patients with IDDVT received either oral anticoagulants or LMWH/fondaparinux, respectively, compared with 73 and $25 \%$ of those with PDVT. ${ }^{13}$ The use of LMWH or fondaparinux presumably reflects a shorter-term anticoagulation strategy for patients with IDDVT, as median duration of anticoagulation was 70 days in the IDDVT group compared with 238 days in the PDVT group. ${ }^{13}$ Notably, our results from RE-COVERY DVT/PE showed that $99.3 \%$ of enrolled patients received anticoagulant therapy. Similarly, in other real-world studies, most IDDVT patients were treated with anticoagulant therapy (- Table $\mathbf{6}$ ). ${ }^{13,14,16,18}$ This finding demonstrates that, in the real-world setting, the selection of anticoagulant therapy as the initial treatment for IDDVT is not different from that for PDVT \pm DDVT. In particular, the strategy of no treatment, albeit mentioned in guidelines and investigated in randomized clinical trials, is rare. Given the limited evidence from randomized controlled trials for a net benefit of anticoagulation in patients with IDDVT, and given the low propagation rate of IDDVT of up to 10 to $20 \%$, these findings may point to a global, significant anticoagulant overuse in a majority of IDDVT patients. Our data provide a basis for future prospective studies investigating risk factors for IDDVT propagation necessitating anticoagulation, or-vice versa-low risk markers, justifying a wait-and-watch strategy in these patients.

We note potential limitations of the study. All diagnostic and treatment information was reported by the investigators with no central or external review, and not all patients would have been examined with both leg vein imaging and computed tomography pulmonary angiography or a ventilation/ perfusion scan. Thus, the distribution of sites of VTE that we have reported should be interpreted with caution and may not represent the natural history of VTE. By contrast, the study provides a global perspective of how physicians diagnose and treat patients with IDDVT.

\section{Conclusion}

These data from the RE-COVERY DVT/PE study demonstrate that, although differences in patient characteristics, risk factors, and clinical management were identified, the selection of initial anticoagulant therapy for IDDVT is not different from that for PDVT \pm DDVT. Prospective studies should investigate whether, in a global perspective, this is an appropriate use of anticoagulants.
Note

Trial registration number (ClinicalTrials.gov) is NCT025 96230.

\section{Authors' Contributions}

S.S. was responsible for the concept of the secondary analyses presented in this manuscript. W.T. was responsible for the data analysis. All authors were responsible for the study concept and design; interpretation of the data; preparation, review, or approval of the manuscript; revision of intellectual content; and the decision to submit the manuscript for publication.

\section{Conflict of Interest}

S. Schellong has received speaker fees from Bayer HealthCare, Boehringer Ingelheim, Bristol-Myers Squibb, Daiichi Sankyo, GlaxoSmithKline, Sanofi, and LEO Pharma. He has received consultancy fees from Bayer HealthCare, Boehringer Ingelheim, Daiichi Sankyo, GlaxoSmithKline, and Sanofi. W. A. has participated in advisory boards for Bayer, Portola, Aspen, Sanofi, Daiichi Sankyo, and Boehringer Ingelheim, and has received travel or research support from Bayer, Portola, Aspen, Janssen, Sanofi, Daiichi Sankyo, Bristol-Myers Squibb, Pfizer, and Boehringer Ingelheim. I.B.C. has received speaker and/or consultancy fees from Boehringer Ingelheim, Bayer, Daiichi Sankyo, Pfizer, and Amgen. K.H.C. has received speaker fees from Boehringer Ingelheim, Bristol-Myers Squibb, and Pfizer. S. Schulman has received honoraria from Boehringer Ingelheim, Bayer HealthCare, Daiichi Sankyo, and Sanofi, and research support from Boehringer Ingelheim, Baxter, and Octapharma. D.E.S. has received honoraria from Boehringer Ingelheim, Bristol-Myers Squibb, Merck, Johnson \& Johnson, and Pfizer, and research support from Boehringer Ingelheim and Bristol-Myers Squibb. M. D., W.T., I.V., and K.Z. are employees of Boehringer Ingelheim. S.Z.G. has received research support from Boehringer Ingelheim, Bristol-Myers Squibb, BTG EKOS, Daiichi Sankyo, Janssen, the US National Heart Lung and Blood Institute, and the Thrombosis Research Institute. He is a consultant for Bayer and Boehringer Ingelheim.

\section{Acknowledgments}

The RE-COVERY DVT/PE study was sponsored by Boehringer Ingelheim. Medical writing assistance, supported financially by Boehringer Ingelheim, was provided by Keith Day, PhD, of Parexel during the preparation of this article. The authors would like to thank all the patients who participated in this study.

\section{References}

1 Beckman MG, Hooper WC, Critchley SE, Ortel TL. Venous thromboembolism: a public health concern. Am J Prev Med 2010;38(4, Suppl):S495-S501

2 Palareti G, Cosmi B, Lessiani G, et al. Evolution of untreated calf deep-vein thrombosis in high risk symptomatic outpatients: the blind, prospective CALTHRO study. Thromb Haemost 2010;104 (05):1063-1070 
3 Palareti G, Schellong S. Isolated distal deep vein thrombosis: what we know and what we are doing. J Thromb Haemost 2012;10(01): 11-19

4 Righini M, Paris S, Le Gal G, Laroche JP, Perrier A, Bounameaux H. Clinical relevance of distal deep vein thrombosis. Review of literature data. Thromb Haemost 2006;95(01):56-64

5 Robert-Ebadi H, Righini M. Should we diagnose and treat distal deep vein thrombosis? Hematology (Am Soc Hematol Educ Program) 2017;2017(01):231-236

6 Schellong SM. Distal DVT: worth diagnosing? Yes. J Thromb Haemost 2007;5(Suppl 1):51-54

7 Cogo A, Lensing AW, Koopman MM, et al. Compression ultrasonography for diagnostic management of patients with clinically suspected deep vein thrombosis: prospective cohort study. BM] 1998;316(7124):17-20

8 Philbrick JT, Becker DM. Calf deep venous thrombosis. A wolf in sheep's clothing? Arch Intern Med 1988;148(10):2131-2138

9 Kearon C, Akl EA, Ornelas J, et al. Antithrombotic therapy for VTE disease. Chest 2016;149(02):315-352

10 Mazzolai L, Aboyans V, Ageno W, et al. Diagnosis and management of acute deep vein thrombosis: a joint consensus document from the European Society of Cardiology working groups of aorta and peripheral vascular diseases and pulmonary circulation and right ventricular function. Eur Heart J 2018;39(47): 4208-4218

11 Ageno W, Casella IB, Han CK, et al. RE-COVERY DVT/PE: rationale and design of a prospective observational study of acute venous thromboembolism with a focus on dabigatran etexilate. Thromb Haemost 2017;117(02):415-421

12 Goldhaber SZ, Ageno W, Casella IB, et al. Profile of patients diagnosed with acute venous thromboembolism in routine clinical practice: the RE-COVERY DVT/PE study. Am J Med 2020;133(08):936-945

13 Barco S, Corti M, Trinchero A, et al. Survival and recurrent venous thromboembolism in patients with first proximal or isolated distal deep vein thrombosis and no pulmonary embolism. J Thromb Haemost 2017;15(07):1436-1442

14 Galanaud JP, Quenet S, Rivron-Guillot K, et al; RIETE Investigators. Comparison of the clinical history of symptomatic isolated distal deep-vein thrombosis vs. proximal deep vein thrombosis in 11 086 patients. J Thromb Haemost 2009;7(12):2028-2034

15 Galanaud JP, Sevestre-Pietri MA, Bosson JL, et al; OPTIMEV-SFMV Investigators. Comparative study on risk factors and early outcome of symptomatic distal versus proximal deep vein thrombosis: results from the OPTIMEV study. Thromb Haemost 2009;102 (03):493-500

16 Schellong SM, Goldhaber SZ, Weitz JI, et al. Isolated distal deep vein thrombosis: perspectives from the GARFIELD-VTE registry. Thromb Haemost 2019;119(10):1675-1685

17 Rothman KJ. A show of confidence. N Engl J Med 1978;299(24): 1362-1363

18 Galanaud JP, Sevestre MA, Pernod G, et al. Long-term outcomes of cancer-related isolated distal deep vein thrombosis: the OPTIMEV study. J Thromb Haemost 2017;15(05):907-916

19 Galanaud JP, Sevestre MA, Genty C, et al; OPTIMEV-SFMV Investigators. Incidence and predictors of venous thromboembolism recurrence after a first isolated distal deep vein thrombosis. J Thromb Haemost 2014;12(04):436-443

20 Barco S, Klok FA, Mahé I, et al; RIETE Investigators. Impact of sex age, and risk factors for venous thromboembolism on the initial presentation of first isolated symptomatic acute deep vein thrombosis. Thromb Res 2019;173:166-171

21 Galanaud JP, Bosson JL, Quéré I Risk factors and early outcomes of patients with symptomatic distal vs. proximal deep-vein thrombosis. Curr Opin Pulm Med 2011;17(05):387-391

22 Schulman S, Kakkar AK, Goldhaber SZ, et al; RE-COVER II Trial Investigators. Treatment of acute venous thromboembolism with dabigatran or warfarin and pooled analysis. Circulation 2014;129 (07):764-772 